\title{
Disruption Management Optimization for Military Logistics
}

\author{
Ayda Kaddoussi ${ }^{1}$, Nesrine Zoghlami ${ }^{1}$, Hayfa Zgaya ${ }^{2}$, \\ Slim Hammadi ${ }^{1}$, and Francis Bretaudeau ${ }^{3}$ \\ ${ }^{1}$ Ecole Centrale de Lille, Cité Scientifique -BP 48, 59651, \\ Villeneuve d'Ascq, France \\ ${ }^{2}$ ILIS, 42, rue Ambroise Paré, 59120 - LOOS France \\ ${ }^{3}$ Logistics Department EADS DS Systems, Saint Quentin en Yvelines, France \\ \{ayda.kaddoussi, nesrine.zoghlami, slim.hammadi\}@ec-lille.fr, \\ hayfa.zgaya@univ-lille2.fr, \\ francis.bretaudeauleads.com
}

\begin{abstract}
To ensure long-term competitiveness, companies try to maintain a high level of agility, flexibility and responsiveness. In many domains, hierarchical SCs are considered as dynamic systems that deal with many perturbations. In this paper, we handle a specific type of supply chain: a Crisis Management Supply Chain (CMSC). Supply during peacetime can be managed by proactive logistics plans and classic supply chain management techniques to guaranty the availability of required needs. However, in case of perturbations (time of war, natural disasters...) the need for support increases dramatically and logistics plans need to be adjusted rapidly. Subjective variables like risk, uncertainty and vulnerability will be used in conjunction with objective variables such as inventory levels, delivery times and financial loss to determine preferred courses of action.
\end{abstract}

Keywords: Logistics, supply chain, risk management, multi-agent.

\section{Introduction}

There is an urgent need for assuring the defense and support mission in time of crisis with a reasonable financial cost. The establishment of an efficient risk assessment that identifies and mitigates deficiencies that could impact mission success must be developed. In the military, decisions are the means by which the commander or decision maker translates their vision of the end state into actions [1]. Hence, there is a need to have the relevant facts and information to make the more adequate and prudent decisions. Experts in all specialties, including logistics, intelligence, medical, etc. deliberate to develop different possible courses of actions and scenarios, and determine what the most likely solution based on their judgment and the feedback from the risk management module and the CMSC in general.

In this paper, a multi-agent based system is proposed to manage the risk induced by the complexity of the CMSC environment. The reminder of the paper is organized as follows: in the following section, definitions about risk and uncertainty are presented, and the usefulness of a multi-agent based framework for SCs risk 
management is discussed. The third section presents a general description of our context and problematic. Then the disruption management process is detailed in section 4. Conclusion and possible future works are addressed in last section.

\section{Literature Review}

Risk can be described as the product of the probability of an event occurring and its consequences, in other words, risk is about uncertainty and its impact [2].Uncertainty is the general property of non-deterministic environment. It generates events that disrupt the pre-established behavior of any complex system such as Supply Chains (SCs). Uncertainty raises risk which can be identified, analyzed, controlled and regulated.

There are different types of disturbances that can be divided to purposeful disturbances (such thefts, terrorism, piracy and financial misdeeds) and nonpurposeful disturbances (such demand fluctuations and bullwhip-effect). The deviations from the expected outcome may affect operations, processes, plans, goals or strategies and would manifest as under achievement of performance, poor reliability, time delay or financial loss. Therefore corrective measures need to be taken to adjust the SC.

Different methods are used for risk management in supply chain. Table 1 summarizes the different methods.

Table 1. Risk management methods

\begin{tabular}{|c|c|}
\hline \multicolumn{2}{|c|}{ Retrospective approaches } \\
\hline Methods & Description \\
\hline Pareto chart & $\begin{array}{l}\text { Used during the analysis phase of reported undesirable events, it } \\
\text { prioritizes the relative importance of different events and ranks them by } \\
\text { decreasing frequency. }\end{array}$ \\
\hline Ishikawa diagram & $\begin{array}{l}\text { It is a causal diagram that shows the causes of a certain event. Each cause } \\
\text { or reason for imperfection is a source of variation. Causes are usually } \\
\text { grouped into major categories to identify these sources of variation. The } \\
\text { categories typically include People, Methods, Machines, Materials and } \\
\text { Environment. }\end{array}$ \\
\hline \multicolumn{2}{|c|}{ Predictive approaches } \\
\hline Methods & Description \\
\hline $\begin{array}{l}\text { FMECA (Failure } \\
\text { Mode, Effects, and } \\
\text { Criticality } \\
\text { Analysis) }\end{array}$ & $\begin{array}{l}\text { It is a procedure for analysis of potential failure modes within a system } \\
\text { by classification of the severity and likelihood of the failures. A } \\
\text { successful FMECA activity helps to identify potential failure modes } \\
\text { based on past experience with similar products or processes, enabling the } \\
\text { team to design those failures out of the system with the minimum of } \\
\text { effort and resource expenditure, by reducing development time and } \\
\text { costs. }\end{array}$ \\
\hline $\begin{array}{l}\text { HACCP (Hazard } \\
\text { Analysis Critical } \\
\text { Control Point) }\end{array}$ & $\begin{array}{l}\text { Aims to assess potential hazards on a given process, and identify } \\
\text { appropriate preventive measures and required monitoring system. Its } \\
\text { application is mostly in catering and food industry. }\end{array}$ \\
\hline $\begin{array}{l}\text { HAZOP ( HAZard } \\
\text { and OPerability } \\
\text { study) }\end{array}$ & $\begin{array}{l}\text { It was initially developed to analyze chemical process systems, but has } \\
\text { later been extended to other types of systems and also to complex } \\
\text { operations and to software systems, to identify and evaluate problems } \\
\text { that may represent risks to personnel or equipment. }\end{array}$ \\
\hline
\end{tabular}


There is an urgent need for SCs planners to have specific strategies to manage these disruptions. Multi-agent technology is very well suited as a technological platform for supporting SC concepts through modeling the different heterogeneous and linked entities. In computer science, an agent can be defined as a software entity, which is autonomous to accomplish its designed goals through the axiom of communication and coordination with other agents [4]. These agents interact and cooperate in order to solve problems beyond their individual knowledge or expertise, and to promote a higher performance for the entire system [5]. Through their learning capability, MAS can demonstrate efficiently the proactive and autonomous behavior of the actors to mitigate disruptions and rectify the SC functioning in real time [6-7]. Agent based technology has also been used for the management of disruptions within a supply chain in some studies. Kimbrough et al. [8], for example, use it for the reduction of the bullwhip effect through modeling a SC with agents. Bansal et al.[9] provide a generalized collaborative framework for risk management oriented to refinery SCs.

\section{Problem Description and Objectives}

The presented work proposes to support the logistics planners in dealing with events that may disrupt the CMSC and get the plan deviate from its intended course. In the case of distributed systems such SCs, this implies a design that unsure an efficient disturbance handling and relevant deployment of individual recovery behaviors. The aim of our approach is to help the different actors of the CMSC to improve their performance and to minimize the impact of disruptions on the whole SC. Our objective are to determine how to deploy the military units and its associated resources and equipment, including personnel, vehicles, and aircraft; and sustain them throughout the operation, by providing supplies (food, water, fuel, etc.) and meeting other needs, such as medical support. Military logistics is a complex process, involving collaboration and coordination among many organisational and informational entities, such as supply, transport and troupes, which are geographically distributed and contain complex information. The Optimisation Based on Agents' Communication (OBAC) is a demonstrator aimed at developing a support system for military logistic that automates the functioning of the military supply chain under crisis. OBAC is prototyped by our LAGIS ${ }^{1}$ research team in collaboration with the Logistics department of EADS, and is investigating, developing and demonstrating technologies to make a fundamental improvement in logistics planning. Agent-based technology has already been applied to different areas in military logistics.

\section{Disruption Management Process}

The risk management module is composed of a set of agents called Disruption Manager Agent (DMA). It is constituted of a watch_agent responsible for providing

${ }^{1}$ LAGIS: Laboratoire d'Automatique, Génie Informatique et Signal. 
monitoring information. It has the ability to trigger an alarm when a disruption event appears. For example, it provides supervision of the delivery process, by monitoring the actual delivery time and comparing it to the planned one. A second agent involved in the DMA is the proposer_rectifying_agent responsible for the suggestion of corrective actions for the emerging risks, based on the alert triggered by the watch_agent. This initiates a process that intents to eliminate or reduce the prominent risk. Other agents will intervene, and will help to facilitate exchange of information and resources, as Zone Agent (ZA: each zone of our CMSC is represented by an agent), GUI Agent (Interface agent), Weather Agent (WA), Need Estimating Agent (NEA), Posts Coordinator Agent (PCA) and Consumption Agent (CA).

\subsection{Risk Identification Phase}

To identify risks, we use quantitative models, and for each activity, risk sources are listed and described. This stage is based on the monitoring of what we called performance indicators (PIs) related to the performance of all actors in the CMSC. The level of in-stock inventory, the amount of resources consumed and delivery dates are some of the PIs that can be used to identify abnormal situations that may engender risks and deficiencies on the CMSC. Actual values of the PIs are monitored by the watch_agent which compares them to predefined values. In case of a significant violation, watch_agent triggers an alarm characterizing the type of violation detected.

\subsection{Risk Assessment Phase}

This phase analyses the impact of the risk identified and provides information about the parameters to be corrected during the decision and selection of the strategy to adopt. To do that, the proposer_rectifier_agent, using the FMEC analysis and based on a risk rating mechanism, estimates the probabilities of occurrence of the event (very unlikely, improbable event, moderate event, probable event, very probable), the risk impact (no impact, minor impact, medium impact or serious impact), and probability of risk non-detectability (very low, low, moderate, high, very high). This assessment is generally based on historical data, experience and advice from experts. The monitoring of the PIs by the watch-agent is maintained in order to provide updates to the DMA. During this step, the success of past decisions applied to the same risk is taken into account for evaluation.

\subsection{Decision and Selection of Corrective Actions Phase}

During this phase, the proposer_rectifier_agent proposes the corrective action to perform for the identified risk. The selected action or strategy is transferred to the agent concerned by the emerging risk to execute it. An example of the behavior of $Z A$ is illustrated by the diagram of activity of figure 1 . 


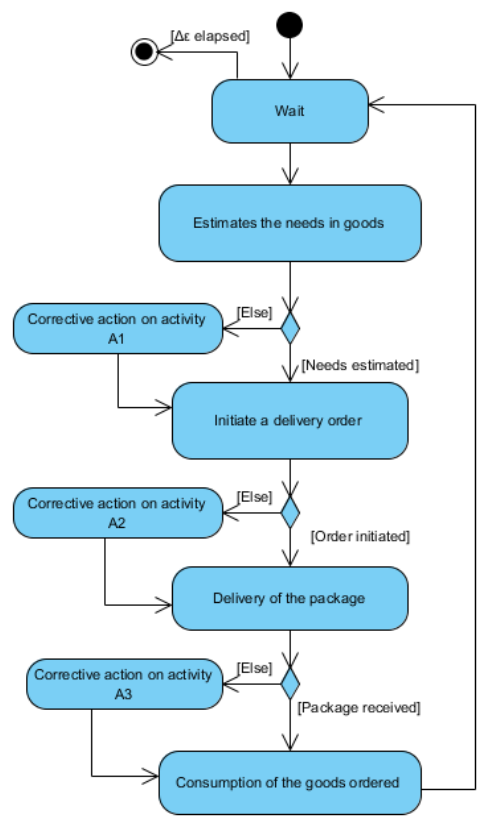

Fig. 1. Behavior of the ZA

\begin{tabular}{|l|l|l|}
\hline Activity & Risk & Corrective Action \\
\hline A1: Need estimation & Inaccurate estimation & Prevent variation with safety stock. \\
\hline A2: Order delivery & Error in the command. & Change the mean of transportation. \\
\hline A3: Order reception & Delay in reception. & Initiate an emergency order. \\
\hline
\end{tabular}

\subsection{Evaluation Phase}

In the final step of the risk management process, the global supply chain performance is evaluated by calculating the avoided cost. This cost is composed of the amount of financial loss that the disruption could have engendered if it had becomes a reality and the investment cost to mitigate the risk. The aim is to get the CMSC efficiency at a higher level or to maintain it at the same level.

This approach is based on a simulation tool which enables the implementation of different models for the planning activities of each actor of the CMSC and takes into account interactions between them.

\section{Validation}

We are developing our system, with JADE platform (Java Agent Development platform) [10]. It is a middleware which permits a flexible implementation of multiagents systems and offers an efficient transport of ACL (Agent Communication Language) messages for agents' communication. To demonstrate the reliability and the applicability of our framework, we can assume a scenario in which the actual 
in-stock inventory is lower than what is indicated by the warehouse manager interface. The situation suggests a delay risk for delivering a specific order to one $Z A$, and as a result the watch-agent triggers a corresponding alarm. This alarm initiates the process to remedy the situation, in which the proposer_rectifier_agent proposes to change the mean of transport as a corrective action. This action is then evaluated and executed if it has been proved to be the optimal one. Else, the proposer_rectifier_agent notifie the ZA and PIs are recalculated in the perspective of performing another corrective action (for instance rationing the consumption on the $Z A$ until the reception of the order).

\section{Conclusion}

This paper has presented a general agent-based framework for disruption management aiming at minimizing the effects of perturbations and uncertainties on a highly distributed crisis management supply chain. The developed process generates courses of actions to have the most effective response to the emergence of a risk. As this work is based on validated models and methods and already existent software tool (OBAC), the next steps consist on the prototyping of a warehouse managing system based on RFID tags in order to track down inventories and so insure better reactivity in a distributed $\mathrm{SC}$.

\section{References}

1. Field Manual - FM No. 101-5, Staff organization and operations contents. Headquarters, Department of the army, Washington, DC, May 31 (1997)

2. Hull, K.: Risk analyses techniques in defense procurement. In: The IEEE Colloquium on Risk Analysis Methods and Tools, London, June 3, pp. 3/1-317 (1992)

3. Giannakis, M., Louis, M.: A multi-agent based framework for supply chain risk management. Journal of Purchasing \& Supply Management 17, 23-31 (2011)

4. Stone, P., Veloso, M.: Multiagent systems: A survey from a machine learning perspective. Autonomous Robots 8(3), 345-383 (2000)

5. Kwon, O., Im, G.P., Lee, K.C.: MACE-SCM: a multi-agent and case-based reasoning collaboration mechanism for supply chain management under supply and demand uncertainties. Expert Systems with Applications 33, 690-705 (2007)

6. Lu, L., Wang, G.: A study on multi-agent supply chain framework based on network economy. Computers and Industrial Engineering 54(2), 288-300 (2007)

7. Kimbrough, S.O., Wu, D.J., Zhong, F.: Computers play the beer game: can artificial agents manage supply chains? Decision Support Systems 33(3), 323-333 (2002)

8. Bansal, M., Adhitya, A., Srinivasan, R., Karimi, I.A.: An online decision support framework for managing abnormal supply chain events. Computer-aided Chemical Engineering 20, 985-990 (2005)

9. Java Agent DEvelopment framework, http://jade.titlab.com/doc 